\title{
Importância do teste de genotipagem em pessoas vivendo com HIV no Estado do
}

\section{Pará, Brasil}

\author{
Importance of the genotypic testing in people living with HIV in the State of Pará, Brazil \\ Importancia de la prueba de genotipación en personas que viven con VIH en el Estado de Pará, \\ Brasil
}

\author{
Aline Cecy Rocha de Lima \\ ORCID: https://orcid.org/0000-0001-5887-3689 \\ Universidade Federal do Pará, Brasil \\ E-mail: alinececy@yahoo.com \\ Alexandre Augusto Bentaberry Rosa \\ ORCID: https://orcid.org/0000-0002-0100-6880 \\ Universidade Federal do Pará, Brasil \\ E-mail: rosa.alexandref@gmail.com \\ Maria Amélia de Oliveira da Costa \\ ORCID: https://orcid.org/0000-0002-3448-1960 \\ Laboratório Central do Pará, Brasil \\ E-mail: maria.ameliadacosta@hotmail.com \\ Rubens Einar Corrêa Dantas \\ ORCID: https://orcid.org/0000-0001-8646-0494 \\ Laboratório Central do Pará, Brasil \\ E-mail: rubenseinar@hotmail.com \\ Andrea Nazaré Monteiro Rangel da Silva \\ ORCID: https://orcid.org/0000-0002-9119-9953 \\ Universidade Federal do Pará, Brasil \\ E-mail: andrearangel@ufpa.br \\ Jacqueline Cortinhas Monteiro \\ ORCID: https://orcid.org/0000-0002-9985-7906 \\ Universidade Federal do Pará, Brasil \\ E-mail: jacqueline@ufpa.br \\ Rosimar Neris Martins Feitosa \\ ORCID: https://orcid.org/0000-0003-2044-9350 \\ Universidade Federal do Pará, Brasil \\ E-mail: rosimar@ufpa.br
}

\begin{abstract}
Resumo
O presente estudo objetivou caracterizar o perfil clinico-epidemiológico de pessoas vivendo com HIV (PVHIV) residentes no estado do Pará. Foram incluídos todos os indivíduos, independente de idade ou sexo, cujas amostras, de 2013 a 2015, foram encaminhadas ao Laboratório Central do Pará (LACEN-PA) para a realização do teste de genotipagem do HIV. Os dados foram obtidos por meio da coleta de informações nos laudos do teste de genotipagem guardados no LACEN. Foram coletadas informações como sexo, idade, nível de escolaridade, raça, ano de diagnóstico, motivos relacionados a mudança de esquema terapêutico e presença de mutações. $\mathrm{O}$ gênero masculino foi predominante, com faixa etária entre 40 e 50 anos ou mais, pardos e com ensino fundamental. A maioria teve o diagnóstico para o HIV entre os anos 2000 e 2009, tendo a maior parte apresentado mudanças de esquema terapêutico. Tal fato se deu em grande parte pela presença de falha terapêutica e intolerância, corroborando assim com a presença de mutações. No presente estudo observamos um perfil epidemiológico de PVHIV condizente com a literatura, caracterizado pela prevalência do gênero masculino, com faixa etária entre 40 e 50 anos ou mais, pardo e escolaridade em nível fundamental. Foi possível observar também um alto índice de mudanças nos regimes terapêuticos, de falha terapêutica, além de elevadas taxas de mutações. Demonstrando assim, a importância do exame de genotipagem não só para a escolha da terapia mais adequada como também para a adoção de estratégias que busquem melhorar a adesão de PVHIV ao tratamento com antirretrovirais. Novos estudos que possam contribuir com a compreensão e identificação das mudanças dos regimes terapêuticos assim como a contribuição destes para o aparecimento de mutações e consequente falha terapêutica são necessários.
\end{abstract}

Palavras-chave: HIV; PVHIV; Genotipagem; Mutações. 


\begin{abstract}
The present study aimed the characterization of the clinical-epidemiological profile of people living with HIV(PVHIV) living in the state of Pará. We included all people, independent of age or sex, whose samples was sent to the Central laboratory of Pará (LACEN-PA) during 2013 to 2015 for the genotyping HIV testing. The data were obtained through the collection of information in the results reports stored in LACEN-PA. Information were collected as sex, age, level of education, race, year of diagnosis, raison related to the change in therapeutic regimen and presence of mutations. The male gender was predominant, aged between 40 and 50 years or more, brown and with elementary education. Most were diagnosed with HIV between the years 2000 and 2009, with the majority showing changes in the therapeutic regimen. This fact was largely due to the presence of therapeutic failure and intolerance, thus corroborating the presence of mutations. In this present study, we observed an epidemiological profile of PLHIV consistent with the literature, characterized by the prevalence of the male gender, with the age group between 40 and 50 years or more, mixed race and education at the fundamental level. It was also possible to observe a high rate of changes in therapeutic regimens, of therapeutic failure, in addition to high rates of mutations for the pharmacological classes used in antiretroviral therapy. Thus, demonstrating the importance of genotyping exams not only for choosing the most appropriate therapy but also for adopting strategies that seek to improve PLHIV adherence to treatment with antiretrovirals. Further, new studies contributing to understand and to identificate the changes in therapeutic regimens, as well as their contribution to the appearance of mutations and consequent therapeutic failure, are needed.
\end{abstract}

Keywords: HIV; PVHIV; Genotypic testing; Mutations.

\title{
Resumen
}

El presente estudio tuvo como objetivo caracterizar el perfil clínico y epidemiológico de las personas que viven con el VIH (PVVIH) residentes en el estado de Pará. Se incluyeron todas las personas, independientemente de su edad o sexo, cuyas muestras de 2013 a 2015 fueron enviadas al Laboratorio Central de Pará (LACEN-PA) para la prueba de genotipado del VIH. Los datos se obtuvieron a través de la recolección de información en los resultados de la prueba de genotipado almacenados en LACEN. Se recogió información como sexo, edad, nivel de estudios, raza, año de diagnóstico, motivos relacionados con el cambio de esquema terapéutico y presencia de mutaciones. Predominó el sexo masculino, con participantes entre 40 e 50 años ou màs, de color marrón y con educación primaria. La mayoría fueron diagnosticados con VIH entre los años 2000 y 2009, y la mayoría mostró cambios en el régimen terapéutico. Este hecho se debió en gran parte a la presencia de fracaso terapéutico e intolerancia, corroborando así la presencia de mutaciones. En el presente estudio observamos un perfil epidemiológico de PVVIH consistente con la literatura, caracterizado por la prevalencia del género masculino, con edad entre 40 y 50 años o más, mestizo y educación a nivel fundamental. También fue posible observar una alta tasa de cambios en los regímenes terapéuticos, de fracaso terapéutico, además de altas tasas de mutaciones. Demostrando así la importancia de los exámenes de genotipificación no solo para elegir la terapia más adecuada sino también para adoptar estrategias que busquen mejorar la adherencia de las PVVIH al tratamiento con antiretrovirales. Se necesitan nuevos estudios que puedan contribuir a la comprensión e identificación de los cambios en los regímenes terapéuticos, así como su contribución a la aparición de mutaciones y el consecuente fracaso terapéutico.

Palabras clave: HIV; PVVIH; Genotipado; Mutaciones.

\section{Introdução}

No mundo, a Síndrome da imunodeficiência adquirida (AIDS) promoveu um importante impacto epidemiológico. Ao longo de 40 anos, cerca de 77,3 milhões de pessoas se infectaram com o vírus da imunodeficiência humana (HIV) e 35,4 milhões morreram de AIDS (Seyler Lucie \& Allard, 2018). A Organização Mundial de Saúde (OMS) estimou que em dezembro de 2019 cerca de 38 milhões de pessoas viviam com HIV, sendo que 690.000 morreram de causas relacionadas ao vírus em 2019. Ainda segundo a OMS cerca de 1,7 milhões de pessoas foram recentemente infectadas (WHO, 2019).

No Brasil o quadro epidemiológico é alarmante, de 2007 a junho de 2020 foram notificados ao Sistema de informação de agravos de notificação (SINAN) cerca de 342.459 casos de infecção pelo HIV no país, sendo a região sudeste a de maior prevalência $(44,4 \%)$ e a região centro-oeste a de menor (7,6 \%). No ano de 2020, foram confirmados no Brasil 13.677 novos casos até o mês de junho, com números mais elevados em homens (69,4\%) do que em mulheres (30,6\%), apresentado assim uma razão de 26 homens para cada 10 mulheres infectadas (BRASIL, 2020).

A terapia antirretroviral (TARV) representou um marco no avanço na luta contra o HIV/AIDS e continua sendo uma estratégia de alto impacto no tratamento ao HIV. No Brasil, a terapia se inicia com uma tripla combinação de 2 Inibidores da transcriptase reversa nucleosídicos (ITRN) associados a uma terceira classe que podem ser os Inibidores da Transcriptase 
Reversa Não Nucleosídicos (ITRNN), os Inibidores da Protease com ou sem ritonavir (IP; IP/r) e os inibidores da Integrase (INI). O esquema inicial preferencial no Brasil é composto pela Lamivudina (3TC), Tenofovir (TDF) e Dolutegravir (DTG). Tal combinação promove um efeito na supressão da replicação do vírus, restaurando o estado imunológico e melhorando a sobrevida do indivíduo. Entretanto a expansão dessa terapia teve como consequência o aparecimento de mutações de resistência ao HIV que afetaram a eficácia dos tratamentos farmacológicos, tornando-se, no atual contexto, um problema de saúde pública (Clutter, Jordan, Bertagnolio, \& Shafer, 2016).

A identificação precoce dessas mutações caracteriza-se por ser uma estratégia global contra o vírus, podendo assim adequar os medicamentos que terão uma melhor eficácia na resposta terapêutica (Mbange et al., 2018). Ao ser detectada a falha virológica busca-se realizar o teste de genotipagem para potencializar o esquema terapêutico adequado para o paciente e realizar a detecção de mutações virais ligadas a resistência aos fármacos e adequação assim, do melhor esquema terapêutico (Brasil, 2018). Nesse contexto, o presente trabalho realizou uma pesquisa de caráter transversal com descrição clínica e epidemiológica do perfil de PVHIV, encaminhados ao Laboratório Central do Estado do Pará (LACEN-PA) para realização de teste de genotipagem e correlacionou o número de trocas de medicamentos utilizados na TARV, com a presença ou ausência de mutações.

\section{Metodologia}

\subsection{Tipo e local de estudo}

O estudo realizado caracterizou-se como transversal, descritivo, retrospectivo (Rouquayrol \& Gurgel, 2018), que incluiu PVHIV residentes no Estado do Pará. Foram incluídos todos os indivíduos, independente de sexo e idade, cujas amostras de sangue foram enviadas para o LACEN-PA para serem encaminhadas para o Laboratório de Referência da Fundação de Medicina Tropical Dr. Heitor Vieira Dourado, em Manaus-AM para a realização do exame de genotipagem do HIV, no período de 2013 a 2015, com informações nos laudos que pudessem dar subsídio a presente pesquisa.

\subsection{Obtenção dos dados e aspectos éticos}

Foram analisados os laudos dos resultados de genotipagem de pessoas vivendo com HIV (PVHIV), realizados no período de janeiro de 2013 a dezembro de 2015, os quais se encontravam arquivados sob a guarda do LACEN - PA. Os dados foram coletados por meio da consulta direta aos laudos e ao SISCEL. Foram coletadas informações sociodemográficas como: Gênero, idade, raça, escolaridade e procedência, assim como foram coletadas informações de cunho clínico como: Ano de diagnóstico, motivos relacionados a troca de esquema terapêutico e presença de mutações. Estas informações obtidas, foram armazenadas em um banco de dados para análise. A pesquisa foi aprovada pelo Comitê de Ética em Pesquisa (CEP) da Universidade Federal do Pará - UFPA, sob o parecer 2.704.658.

A análise estatística dos dados foi realizada utilizando-se o programa estatístico Graphpad prism, versão 3.0.1. Utilizou-se o teste de Qui-quadrado para a comparação de frequências e a correlação realizada baseou-se no teste de correlação de Friedman. O nível de significância adotado em todos os testes foi de 5\% (p-value < 0,05).

\section{Resultados}

Um total de 189 laudos de teste de genotipagem do HIV, realizados durante o período de 2013 a 2015 foram avaliados. As análises demonstraram que 52,91\% eram homens e 47,09\% mulheres. Em relação a idade, a faixa etária de 36 a 65 anos apresentou a maior prevalência. 34,92\% declararam ser pardos seguidos da raça negra (11,64\%). Observou-se que a ausência de dados referentes a raça foi elevada (44,97\%). O nível de escolaridade com maior predominância foi o Ensino 
fundamental $(20,11 \%)$. Entretanto a ausência de informações em relação a escolaridade encontrada nesse estudo também foi elevada $(59,26 \%)$ (Tabela 1$)$.

Tabela 1. Dados epidemiológicos de pessoas vivendo com HIV-1 in Pará, Brasil no período de 2013 a 2015.

\begin{tabular}{cccc}
\hline Variável & Categoria & N & \% \\
\hline \hline \multirow{2}{*}{ Gênero } & Masculino & 100 & 52,9 \\
& Feminino & 89 & 47,1 \\
\hline \multirow{2}{*}{ Idade } & $<13$ & 9 & 4,8 \\
& $13-19$ & 4 & 2,1 \\
& $20-29$ & 10 & 5,3 \\
& $30-39$ & 43 & 22,8 \\
& $40-49$ & 59 & 31,2 \\
& 50 ou mais & 63 & 33,3 \\
& Não Informado & 1 & 0,5 \\
\hline \multirow{2}{*}{ Raça } & Amarela & 3 & 1,6 \\
& Pardo & 66 & 34,9 \\
& Negro & 22 & 11,6 \\
& Branco & 13 & 6,9 \\
& Não Informado & 85 & 45,0 \\
\hline & Fundamental & 38 & 20,1 \\
& Ensino médio & 29 & 15,3 \\
& Superior & 10 & 5,3 \\
& Não Informado & 112 & 59,3 \\
\hline & & & \\
& & &
\end{tabular}

Fonte: Autores.

A maior parte das PVHIV que realizaram a genotipagem eram provenientes do município de Belém (47\%), seguido do interior com $34 \%$ (que abrange todos os municípios fora de belém e da região metropolitana) e região metropolitana com 16\%. Quando considerados região metropolitana e Belém, a soma abrange 63\% dos casos (Figura 1).

Figura 1. Distribuição do local de residência de PVHIV submetidos ao exame de genotipagem do HIV-1 no estado do Pará, no período de 2013 a 2015.

\section{Procedéncia das PVHIV}
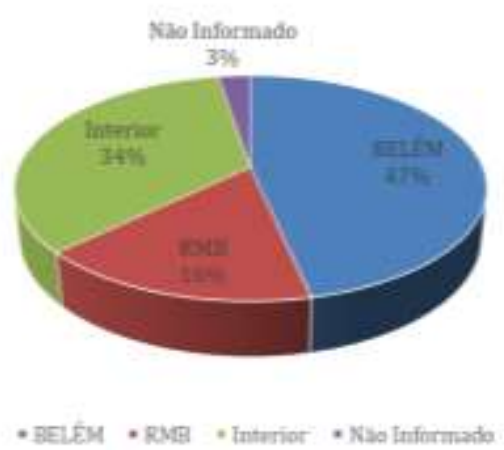

Fonte: Autores. 
A maior parte das PVHIV desse estudo (63\%), foram diagnosticadas entre os anos de 2000 e 2009. Constatou-se que $142(75 \%)$ mudaram de esquema terapêutico, sendo $39 \%$ do gênero masculino e 36\% do gênero feminino. Do total avaliado, 16\% não trocaram de esquema terapêutico, 67\% apresentaram de 1 a 5 trocas e apenas $4 \%$ apresentaram mais de 6 trocas, cerca de 12\% não apresentaram informações em seus prontuários (Tabela 2). Não houve diferença estatística para nenhuma das variáveis entre os grupos analisados.

Tabela 2. Dados clínicos de PVHIV no Pará, Brasil, no período de 2013 a 2015.

\begin{tabular}{|c|c|c|c|c|c|c|}
\hline & & & & & & \\
\hline & & $\mathbf{N}$ & $\%$ & $\mathbf{N}$ & $\%$ & \\
\hline & 1992 a 1999 & 16 & 8 & 7 & 4 & \\
\hline & 2000 a 2009 & 56 & 30 & 63 & 33 & \\
\hline Ano de diagnóstico & & & & & & 0,13 \\
\hline & 2010 a 2014 & 22 & 12 & 13 & 7 & \\
\hline & Não Informado & 6 & 3 & 6 & 3 & \\
\hline & $\mathrm{Sim}$ & 74 & 39 & 68 & 36 & \\
\hline Mudança de esquema & Não & 20 & 11 & 12 & 6 & 0,45 \\
\hline & Não Informado & 6 & 3 & 9 & 5 & \\
\hline & 0 & 19 & 10 & 12 & 6 & \\
\hline Quantas vezes houve mudança & 1 a 5 & 65 & 34 & 62 & 33 & \\
\hline de esquema terapêutico & 6 a 9 & 4 & 2 & 4 & 2 & \\
\hline & Não Informado & 11 & 6 & 11 & 6 & \\
\hline
\end{tabular}

Legenda: (Sim) Houve mudança de esquema; (Não) Não houve mudança de esquema. Teste do X2 para niveis de significância de p-value (< 0,05). Fonte: Autores.

Os motivos mais comuns que levaram a troca de esquema terapêutico foram: Falha terapêutica ou intolerância (41\%), seguido de não adesão ao tratamento (11\%), gravidez (4\%), reações adversas (1\%), tratamento da tuberculose (1\%) e descontinuação de uma das drogas (1\%). Além disso, em cerca de 80 prontuários (41\%) nao foi encontrada nenhuma informação sobre os motivos referentes a troca de esquema terapêutico (Figura 2). 
Figura 2. Motivos relacionados a mudança de esquema terapêutico em PVHIV no Pará, no periodo de 2013 e 2015.

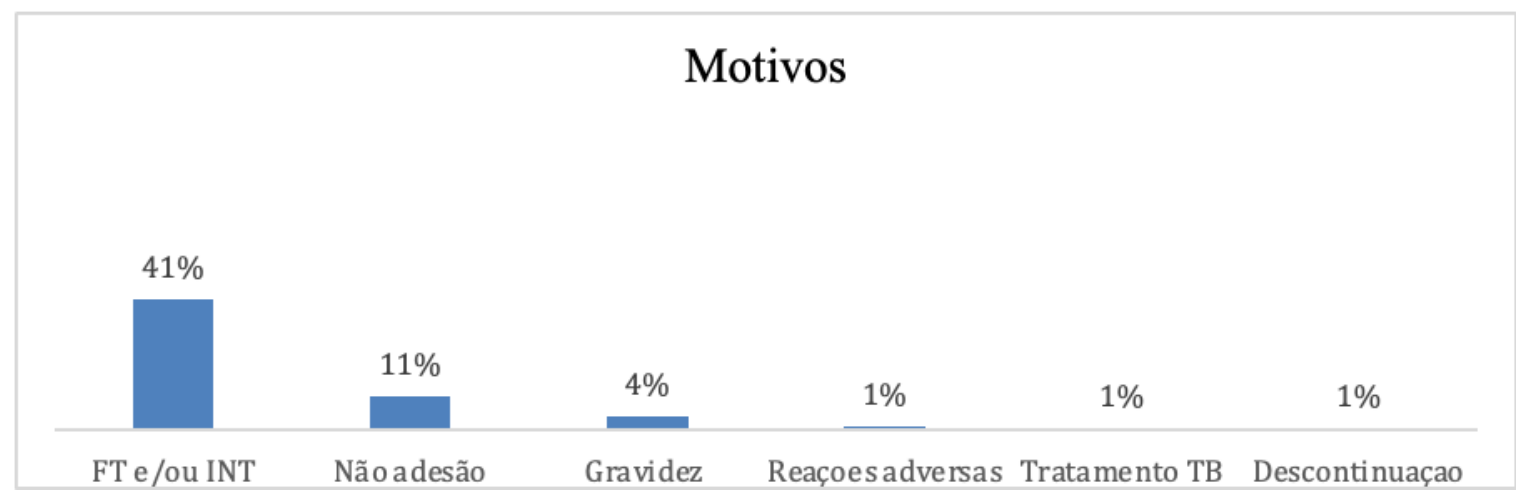

Legenda: FT: Falha terapêutica, INT: Intolerância, TB:Tuberculose. Fonte: Autores.

A análise dos laudos de genotipagem revelou uma alta frequência de mutações associadas às classes farmacológicas: ITRNN (72\%), ITRN (98\%) e PR (99\%). Além disso, foram encontrados 26\% de ausência de informações nos laudos para ITRNN (Figura 3).

Figura 3. Frequência de mutações associadas às classes farmacológicas ITRNN, ITRN e PR em PVHIV no Pará, Brasil, no período de 2013 a 2015.

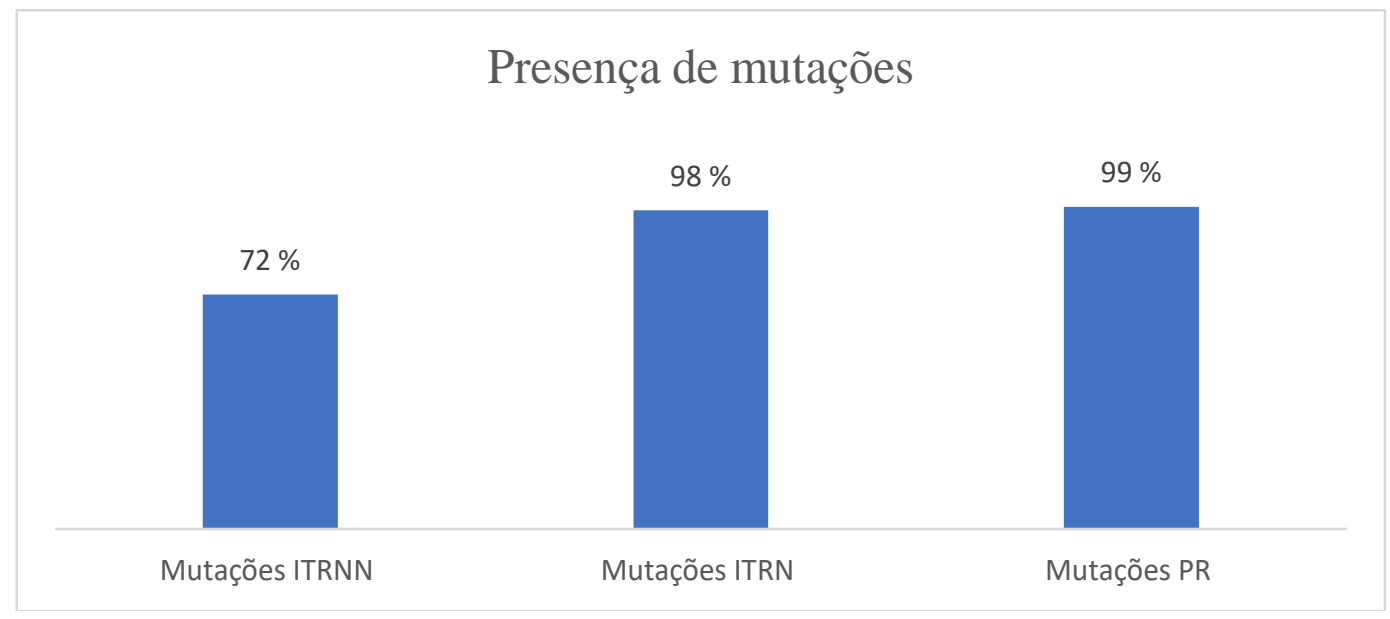

Legenda: ITRNN: Inibidores da transcriptase reversa não nucleosídicos, ITRN: Inibidores nucleosídicos da transcriptase reversa, PR: Inibidores da Protease. Fonte: Autores.

Baseado nos dados encontrados, era esperado que a quantidade de mudanças de esquema terapêutico pudesse estar correlacionada com o surgimento de mutações. Entretanto ao ser utilizado o teste de correlação de Spearman nenhuma correlação foi encontrada ( $\mathrm{p}=0,27)$.

\section{Discussão}

Os achados epidemiológicos do presente estudo corroboram outros estudos realizados, onde a maior prevalência detectada é no gênero masculino (Azevedo, Ximenes, Monteiro, Montarroyos, \& Miranda-Filho, 2020; Rodriguez, Torvisco, \& Aceitero, 2019). Uma possível explicação para essa frequência maior em homens é o fato de o mesmo costumar ser visto pela 
sociedade como sempre um ser que deve estar preparado para o sexo, pois deve comprovar sua virilidade a todo o momento (Domingues, Marcos, Gomes, Cristina, \& Iii, 2016). A faixa etária predominante neste estudo também está de acordo com os estudos realizados anteriormente, os quais demonstram um perfil de PVHIV entre 40 e 50 anos ou mais (Qiao et al., 2019; WHO, 2019).

A coleta de dados referentes a raça pode contribuir nas políticas públicas de prevenção e acompanhamento da TARV. Segundo o último boletim de vigilância do CDC, a maior prevalência em 2018 encontrava-se na população negra / afro americanos, seguida da população hispânica/latinos e brancos (CDC, 2020), tal dado corrobora os dados achados no presente estudo. No Brasil a população negra e parda, compõe a população de baixa renda em geral, a qual tem acesso ao Sistema Único de Saúde (SUS) que se caracteriza por ser gratuito e universal e possui um papel fundamental na redução de desigualdades (Machado, Lima, \& Baptista, 2017). A abordagem racial no contexto do HIV caracteriza-se por ser de suma importância, sabe-se que somente em 1996 em 2001 o Sistema de Informação de Mortalidade (SIM) e o Sistema de Informação de Agravos de Notificação (SINAN), passaram a abordar os dados referentes a raça. Além de recente é ainda hoje deficiente (Fry, Maio, Maio, Bastos, \& Santos, 2007). Outro fator de suma importância a ser abordado é a falta de informações contidas nos laudos haja vista que mais de $40 \%$ dos prontuários não foram preenchidos adequadamente. Tal fato dificulta a coleta de dados e a abordagem correta dos pacientes (Moura, Pinto, Faria, \& Rodrigues, 2017).

A infecção pelo HIV ainda hoje está intimamente ligada ao contexto social, assim sobre o nível de escolaridade os resultados aqui apresentados estão de acordo com o que vem sendo relatado em diversas pesquisas (Bhata, Aryal, \& Khanal, 2013; Gomes, Ceccato, Kerr, \& Guimarães, 2017; Jary et al., 2019; Qiao et al., 2019; Silveira, Santos, Falco, Cardoso, \& Vitorino, 2018), as quais afirmam que quanto maior o nível de instrução mais frequente se torna o uso de preservativo, sugerindo dessa forma uma possível correlação entre baixo nível de escolaridade e suscetibilidade à infecção pelo HIV (Dourado, Maccarthy, Reddy, Calazans, \& Gruskin, 2015). Observou-se neste estudo que a maioria dos casos eram procedentes do município de Belém. Estes dados corroboram estudos realizados em outras cidades do Brasil, os quais demonstraram que as PVHIV apresentaram um perfil mais urbano, tal fato justifica-se pelo início da epidemia ter se dado pelos grandes centros urbanos, além da própria concentração populacional ser mais elevada na mesorregião Metropolitana. Além disso, sugere-se uma possível interiorização da infecção, como já foi observado em outro estudo (Abreu et al., 2016).

Os dados encontrados nesse estudo demonstram um aumento no diagnóstico do HIV apartir dos anos 2000, tal achado reflete as consquistas das estratégias de implementação de centros de testagem e aconselhamento (CTA) nos municípios brasileiros. Segundo o Ministério da Saúde (MS) a estratégia de implementação de CTA se deu a partir dos anos 90; mais precisamente em 1998. Entretanto mesmo que cerca de 430 municípios estivessem dentro da política de incentivo do MS, portanto, prioritários para o controle e acompanhamento de IST/AIDS, cerca de 209 (48,9\%) nao possuíam CTA em 2008 (Brasil, 2008). A presença de falha terapêutica ou intolerância foi detectada em $41 \%$ dos laudos analisados. Na literatura já foi relatada falha virológica oriunda de níveis inadequados de medicamentos, além disso, outra razão que pode colaborar para este fato é a absorção gastrointestinal limitada, assim como uma baixa adesão (McCluskey, Siedner, \& Marconi, 2019). Pacientes recebem diversos esquemas terapêuticos por longos períodos, podendo apresentar cepas resistentes a vários tipos de drogas utilizadas no tratamento anti-HIV. A caracterização da resistência terapêutica, pode se tornar complicada, uma vez que existem uma infinidade de mutações de resistência a essas drogas que se apresentam em padrões complexos levando a níveis variados de resistência medicamentosa (Clutter et al., 2016).

A não adesão ao tratamento pode ser evidenciada como o segundo maior motivo para trocas terapêuticas no presente estudo. Inúmeras razões como: Perfil da doença (crônica ou aguda), status social/econômico e acesso a serviços de saúde podem contribuir para a não adesão (Cea-Calvo et al., 2020). O presente estudo constatou que $67 \%$ dos pacientes realizaram de 1 a 5 mudanças de esquema terapêutico durante o tratamento, essa realidade pode se justificar pela baixa adesão aos esquemas 
terapêuticos, sendo este fato devido em grande parte a toxicidade (Sá Bandeira, Dias Elias, Cavalcante, Lima, \& Távora, 2017). Existe a necessidade que o preescritor assim como as equipes multidisciplinares levem em consideração as características individuais de cada paciente, facilitando assim a adesão e reduzindo os eventos de troca de esquemas, fato este que poderia contribuir a redução do aparecimento de toxicidade e de perfis de resistência (Brites, 2016; Sisay, Edessa, Ayele, \& Getachew, 2019).

Foram encontradas principalmente a presença de mutações relacionadas às classes ITRNN (72\%), ITRN (98\%) e PR (99\%), o que corrobora com alguns estudos realizados no estado do Pará anteriormente (Irias et al., 2019; MACÊDO, DE SOUSA, Ferreira, Vasconcelos, \& Araújo, 2011). Este padrão de resistência pode estar relacionado as cepas virais e subtipos circulantes na região paraense. Além disso, é válido ressaltar que o fámaco Zidovudina (AZT), o qual pertence a classe dos ITRN's foi o primeiro antirretroviral introduzido para o tratamento de PVHIV logo é possível supor que existe a probabilidade de se criar resistência mais rapidamente. Nesse contexto, o aumento de acesso a TARV ao longo do tempo, pode estar promovendo uma pressão seletiva de cepas resistentes aos medicamentos utilizados nos tratamentos para HIV. Apesar das informações descritas por estudos realizados na região norte do Brasil (Pará e Amazonas), estudos que coloquem em evidência dados clínicos (frequências de mutações, perfil de resistência de fármacos) e epidemiológicos ainda são poucos e necessitam de uma atualização, este fato pode ser considerado um fator limitante em níveis de comparações para o nosso estudo. Outra limitação para o nosso estudo foi o fato de a partir do ano de 2016 os testes de genotipagem da rede do MS passarem a ser realizados por outro laboratório, não sendo assim mais possível o acesso aos laudos para a continuidade da pesquisa.

\section{Conclusão}

No presente estudo observamos um perfil epidemiológico de PVHIV condizente com a literatura, caracterizado pela prevalência do gênero masculino, com faixa etária de 50 anos ou mais, pardo e escolaridade em nível fundamental. Foi possível observar também um alto índice de mudanças nos regimes terapêuticos, de falha terapêutica, além de elevadas taxas de mutações para as classes farmacológicas usadas na terapia antirretroviral, demonstrando assim, a importância do exame de genotipagem não só para a escolha da terapia mais adequada como também para a adoção de estratégias que busquem melhorar a adesão de PVHIV ao tratamento com antirretrovirais. Novos estudos que possam contribuir com a compreensão e identificação das mudanças dos regimes terapêuticos assim como a contribuição destes para o aparecimento de mutações e consequente falha terapêutica são necessários.

\section{Agradecimentos}

O presente estudo foi financiado pela Coordenação de Aperfeiçoamento de Pessoal de Nível Superior (CAPES-Brasil). Os autores expressam seus agradecimentos ao Laboratório de Virologia da Universidade Federal do Pará (UFPA) e ao Laboratório Central do Estado do Pará (LACEN-PA).

\section{Referências}

Abreu, S. R. de, Pereira, B. M., Silva, N. M. da, Moura, L. R. P., Brito, C. M. S. de, \& Câmara, J. T. (2016). Estudo epidemiológico de pacientes com infecção pelo virus da imunodeficiencia humana Síndrome da Imunodeficência adquirida (HIVAIDS), CAXIAS-MA. Revista Interdisciplinar, 9(4), $132-141$.

Azevedo, L. N., Ximenes, R. A. de A., Monteiro, P., Montarroyos, U. R., \& Miranda-Filho, D. de B. (2020). Factors associated to modification of first-line antiretroviral therapy due to adverse events in people living with HIV / AIDS. The Brazilian Journal of Infectious Diseases, 24(1), 65-72.

Bhata, D. N., Aryal, U. R., \& Khanal, K. (2013). Education: The key to curb HIV and AIDS epidemic. Kathmandu University Medical Journal, 11(42), 158161. https://doi.org/10.3126/kumj.v11i2.12493

Brasil. (2018). Manejo da infecção pelo hiv em adultos. In Secretaria de Vigilância em Saúde. Departamento de Vigilância, Prevenção e Controle das Infecções Sexualmente Transmissíveis, do HIV/Aids e das Hepatites Virais. Protocolo. 
Brasil, M. da S. (2008). Manual de adesão ao tratamento para pessoas vivendo com HIV e AIDS. In Secretaria de Vigilância em Saúde. Departamento de Vigilância, Prevenção e Controle das Infecções Sexualmente Transmissíveis, do HIV/Aids e das Hepatites Virais. Protocolo.

BRASIL, M. da S. (2020). Boletim Epidemiológico HIV / Aids | 2020. Secretaria de Vigilância Em Saúde, 1, 68.

Brites, C. (2016). Estratégias de troca ( switch ) na terapia antirretroviral atual. Educaçao Medica Continuada, 2.

CDC. (2020). HIV Surveillance Supplemental Report: Estimated HIV Incidence and Prevalence in the United States, 2014-2018. 25(1), 2010-2015.

Cea-Calvo, L., Marín-Jiménez, I., de Toro, J., Fuster-RuizdeApodaca, M. J., Fernández, G., Sánchez-Vega, N., \& Orozco-Beltrán, D. (2020). Association between non-adherence behaviors, patients' experience with healthcare and beliefs in medications: a survey of patients with different chronic conditions. Current Medical Research and Opinion, 36(2), 293-300.

Clutter, D. S., Jordan, M. R., Bertagnolio, S., \& Shafer, R. W. (2016). HIV-1 Drug Resistance and Resistance Testing. Infect Genet Evol, 46(1), 292-307.

Domingues, S., Marcos, A., Gomes, T., Cristina, D., \& Iii, D. O. (2016). Representações sociais de homens sobre o ser homem e suas implicações para o HIV / AIDS. 24(6), 1-6.

Dourado, I., Maccarthy, S., Reddy, M., Calazans, G., \& Gruskin, S. (2015). Revisitando o uso do preservativo no Brasil. Revista Brasileira de Epidemiologia, $18(1), 63-88$.

Fry, P. H., Maio, M. C., Maio, M. C., Bastos, F. I., \& Santos, R. V. (2007). AIDS tem cor ou raça ? Interpretação de dados e formulação de políticas de saúde no Brasil Does AIDS have a race or color? Data interpretation and health policymaking in Brazil. Caderno de Saúde Pública, 23(3), 497-523.

Gomes, R. R. de F. M., Ceccato, M. das G. B., Kerr, L. R. F. S., \& Guimarães, M. D. C. (2017). Fatores associados ao baixo conhecimento sobre HIV/AIDS entre homens que fazem sexo com homens no Brasil. Cadernos de Saude Publica, 33(10), 1-15.

Irias, S. D. F., Torres, M. K. S., Avelino, M. E. S., Santos, M. B., Queiroz, M. A. F., Freitas, F. B. MacHado, L. F. A. (2019). HIV-1 Transmitted Drug Resistance Mutations in Recently Diagnosed Antiretroviral-Naive Patients in Belém, Pará, Northern Brazil. AIDS Research and Human Retroviruses, 35(2), $121-122$

Jary, A., Dienta, S., Leducq, V., Le Hingrat, Q., Cisse, M., Diarra, A. B., ... Maiga, A. I. (2019). Seroprevalence and risk factors for HIV, HCV, HBV and syphilis among blood donors in Mali. BMC Infectious Diseases, 19(1), 1-8.

Macêdo, O., De Sousa, R. C., Ferreira, L. M., Vasconcelos, P., \& Araújo, J. R. (2011). Genotipagem da resistência genotípica secundária aos antirretrovirais em pacientes com aids nos Estados do Pará e Amazonas, Brasil : 2002 a 2006 *. 55(91), 27-34.

Machado, C. V., Lima, L. D. de, \& Baptista, T. W. de F. (2017). Health policies in Brazil in times of contradiction: paths and pitfalls in the construction of a universal system. Cadernos de Saude Publica, 33(2), 144-161.

Mbange, A. E., Kaba, D., Diouara, A. A. M., Diop-Ndiaye, H., Ngom-Ngueye, N. F., Dieng, A., ... Kane, C. T. (2018). Surveillance of transmitted HIV-1 antiretroviral drug resistance in the context of decentralized HIV care in Senegal and the Ebola outbreak in Guinea. BMC Research Notes, $11(1), 723$.

McCluskey, S. M., Siedner, M. J., \& Marconi, V. C. (2019). Management of Virologic Failure and HIV Drug Resistance. Infectious Disease Clinics of North America, 33(3), 707-742.

Moura, Pinto, J., Faria, \& Rodrigues, M. (2017). Caracterização E Perfil Epidemiológico Das Pessoas Que Vivem Com Hiv / Aids Characterization and Epidemiological Profile of People Living With Hiv / Aids. Rev Enferm UFPE, 11, 5214-5220.

Qiao, Y. chao, Xu, Y., Jiang, D. xiang, Wang, X., Wang, F., Yang, J., \& Wei, Y. sheng. (2019). Epidemiological analyses of regional and age differences of HIV/AIDS prevalence in China, 2004-2016. International Journal of Infectious Diseases, 81, 215-220.

Rodriguez, M. C., Torvisco, M. del M. L.-T., \& Aceitero, J.-M. R. (2019). Vigilancia unificada de la infección por vih y enfermedad de sida en extremadura en el período 1980-2018. Rev Espanhola Salud Publica, 93, 1-11.

Rouquayrol, M. Z., \& GURGEL, M. Rouquayrol: epidemiologia e saúde. 8. ed. Rio de Janeiro: MedBook, 2018.719 p.

Sá Bandeira, A. C. P. C., Dias Elias, D. B., Cavalcante, M. G., Lima, D. G. L., \& Távora, L. G. F. (2017). Antiretroviral changes during the first year of therapy. Revista Da Associacao Medica Brasileira, 63(7), 606-612.

Seyler Lucie, L. P., \& Allard, S. D. (2018). Current challenges in the treatment of HIV. Pol Arch Intern Med, 44(0028-3878 LA-eng PT-Journal Article PTReview PT-Review, Tutorial RN-0 (Anticonvulsants) SB-AIM SB-IM), S4-S9.

Silveira, E. A., Santos, A. S. e. A. de C., Falco, M. de O., Cardoso, R. de C., \& Vitorino, P. V. de O. (2018). Association of physical inactivity with hypertension and low educational level in people living with HIV / AIDS. AIDS Care, 30(8), 1004-1009.

Sisay, M., Edessa, D., Ayele, Y., \& Getachew, M. (2019). Pattern of and reasons for antiretroviral therapy regimen change among adult HIV/AIDS patients at regional hospital in Eastern Ethiopia: A 10-year retrospective study. SAGE Open Medicine, 7, 205031211982709.

WHO, W. health organization. (2019). HIV/AIDS. World Health Organization, (September), 78. 